\title{
Neutrons for Science (NFS) at SPIRAL-2
}

\author{
Danas Ridikas \\ CEA Saclay, DSM/DAPNIA \\ 91191 Gif-sur-Yvette, France \\ E-mail: danas.ridikas@cea.fr

\section{Xavier Ledoux ${ }^{\star}$} \\ CEA/DIF, DAM/DPTA \\ 91680 Bruyères-le-Châtel, France \\ E-mail: xavier.ledoux@cea.fr
}

\begin{abstract}
A huge number of high energy neutrons (in the range between 1 and $40 \mathrm{MeV}$ ), produced in the carbon converter via $\mathrm{C}(\mathrm{d}, \mathrm{xn})$ reaction, will be available at the SPIRAL-2 project at GANIL (Caen, France) aiming to produce neutron-rich fission fragments. The facility is expected to be operational by 2010. The main goal of this study is to provide quantitative estimates on the possibility of using the same linear deuteron accelerator $(40 \mathrm{MeV} ; 5 \mathrm{~mA} ; 200 \mathrm{~kW})$ in a combination with the rotating target-converter, as initially projected at SPIRAL-2 for Radioactive Ion Beam (RIB) production, for neutron production, namely, a) material irradiations very close to the target-converter, and b) time-of-flight ( $\mathrm{ToF})$ measurements with a pulsed neutron beams.

In this paper we show that for these two particular applications SPIRAL-2 may become an attractive facility providing a very high intensity neutron source. Some particular requirements and limits are identified in this context.
\end{abstract}

International Workshop on Fast Neutron Detectors University of Cape Town, South Africa

April 3 - 6, 2006

\section{* Speaker}




\section{Introduction}

Material testing via irradiation in high energy and high intensity neutron fluxes is of great interest for very extended community working on nuclear waste transmutation (fast reactors and use of ADS in particular), intensive neutron sources (SNS, J-PARK, IFMIF, ...), radioactive ion beam (RIB) production with neutrons (EURISOL, RIA, ...), future controlled fusion experiments and reactors (ITER, DEMO, ...), space applications (resistance of electronics, shielding, ...), basic nuclear data for high energy neutrons, etc. Our goal is to characterize in more detail the potential of using SPIRAL-2 neutrons for these particular applications.

Indeed, a huge number of high energy neutrons $\left(\sim 10^{15} \mathrm{n} / \mathrm{s}\right.$; in the range between 1 and 40 $\mathrm{MeV})$, produced in the carbon converter via $\mathrm{C}(\mathrm{d}, \mathrm{xn})$ reaction, will be available at the SPIRAL-2 project at GANIL (Caen, France) aiming to produce neutron-rich fission fragments [1]. The main goal of this study is to provide quantitative estimates on the possibility of using a $40 \mathrm{MeV}$ (5mA) linear deuteron accelerator in a combination with a carbon target-converter, as projected at SPIRAL-2, for a) material irradiations, and b) potential time-of-flight experiments with a pulsed neutron beam. We note that the use of this intensive neutron source was already discussed in detail at the SPIRAL-2 workshop, held at GANIL (Caen) France in 2004 [2]. This paper is aiming to summarize these potential possibilities.

\section{Characterization of the neutron source}

The neutrons produced by $\mathrm{d}(40 \mathrm{MeV})+\mathrm{C}$ reaction present a continuous energy spectrum up to $\sim 40 \mathrm{MeV}$ and are peaked at forward angles. All estimates in this work were done using the MCNPX code system [3] by adjusting the total neutron yield with respect to the existing experimental data. The $\mathrm{d}(40 \mathrm{MeV})+\mathrm{C}$ reaction results in 0.025 neutrons per incident deuteron (already corrected) with an average neutron energy around $14 \mathrm{MeV}$ as presented in Fig.1 (on the left). With this reaction rate one obtains $\sim 10^{15} \mathrm{n} / \mathrm{s}$ (with the primary beam intensity of $5 \mathrm{~mA}$ ) emitted mostly in forward angles.

Note that in this case a carbon disk of $1 \mathrm{~cm}$ thick was bombarded by $40 \mathrm{MeV}$ deuteron beam with a $5 \mathrm{~cm}^{2}$ beam spot distributed homogeneously on the sphere. In Fig. 2 (on the right) the calculated neutron flux as a function of distance from the production target is presented (red points). Due to a particularity of ( $\mathrm{d}, \mathrm{xn})$ reaction, the neutron source is not isotropic, and the $\sim 1 / \mathrm{L}^{2}$ law is valid only at distances greater than $200 \mathrm{~cm}$ (blue line).

\section{Material irradiations}

Table 1 provides basic irradiation facility specifications concerning SPIRAL-2 compared to IFMIF [4]. According to these preliminary estimates, it seems that SPIRAL-2 would be able to provide neutron fluxes by a factor of $\sim 10$ lower than IFMIF. In addition, this will be valid only for much smaller irradiation volumes (in a first approximation by a factor of $\sim 10$, i.e. scaled as a ratio between corresponding deuteron beam spots on the target). Another important 
finding is that at forward angles the $\mathrm{d}+\mathrm{C}$ reaction results in a slightly harder neutron spectrum than $\mathrm{d}+\mathrm{Li}$. This is due to a higher contribution of neutron production from the target fragmentation and/or compound nucleus evaporation in the case of $\mathrm{Li}$.
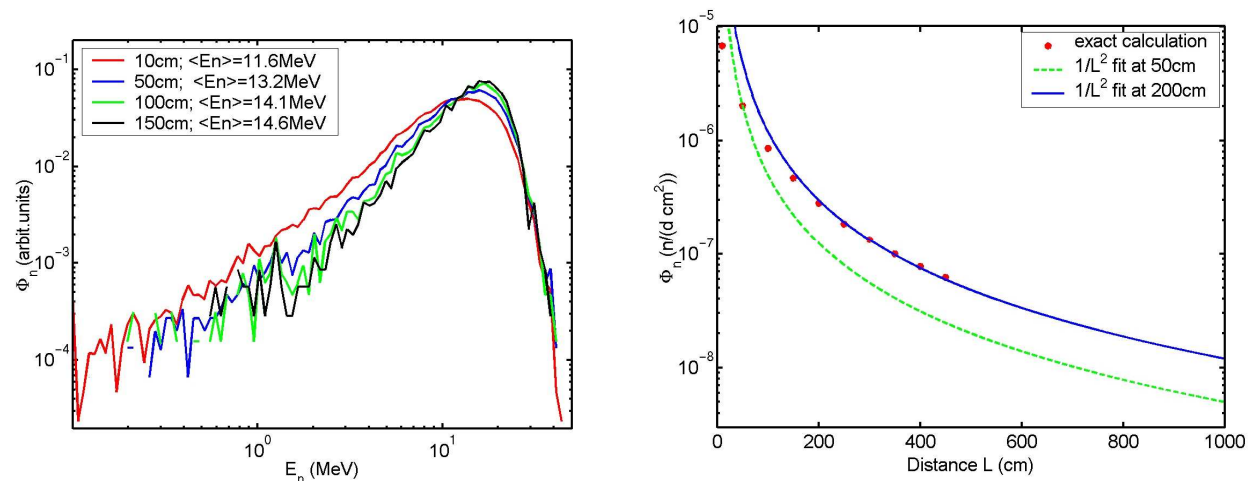

Figure 1: on the left - energy spectra and average neutron energies as a function of the distance from the neutron source towards the deuteron beam direction; on the right-neutron flux as a function of the distance, normalised per incident deuteron. Red points are exact MCNPX calculation results. Blue and green lines are $\sim 1 / L^{2}$ curve scaled to the flux at $200 \mathrm{~cm}$ and $50 \mathrm{~cm}$ respectively.

Table 1: Major expected facility characteristics for SPIRAL-2 and IFMIF.

\begin{tabular}{|l|r|r|}
\hline Project & \multicolumn{1}{|c|}{ IFMIF } & \multicolumn{1}{|c|}{ SPIRAL-2 } \\
\hline Reaction specification & $\mathrm{d}(40 \mathrm{MeV})+\mathrm{Li}$ & $\mathrm{d}(40 \mathrm{MeV})+\mathrm{C}$ \\
\hline Maximum beam current $(\mathrm{mA})$ & $2 \times 125$ & 5 \\
\hline Beam spot on the target $\left(\mathrm{cm}^{2}\right)$ & $\sim 100$ & $\sim 10$ \\
\hline Beam density on the target $\left(\mathrm{mA} / \mathrm{cm}^{2}\right)$ & 2.5 & 0.5 \\
\hline Neutron production over $4 \pi(\mathrm{n} /$ deuteron) & $\sim 0.07$ & $\sim 0.03$ \\
\hline Neutron source intensity $(\mathrm{n} / \mathrm{s})$ & $\sim 10^{17}$ & $\sim 10^{15}$ \\
\hline Maximal neutron flux on the back-plate $\left(\mathrm{n} /\left(\mathrm{s} \mathrm{cm}^{2}\right)\right.$ & $\sim 10^{15}$ & $\sim 12$ \\
\hline$<$ E $_{\mathrm{n}}>$ on the back-plate $(\mathrm{MeV})$ & $\sim 10$ & $\sim 10$ \\
\hline
\end{tabular}

In order to detail the irradiation conditions we consider an irradiation volume (cylinder) filled with ${ }^{56} \mathrm{Fe}$ and ${ }^{4} \mathrm{He}$ with equal occupations (50\%-50\%) and placed right behind the converter target. Partial occupation of the irradiation volume was chosen to allow for possible cooling (typically He-gas flow). The irradiation zone was of cylindrical shape with $6 \mathrm{~cm}$ in length and in diameter (Figure 3; on the left). All calculations were carried out with the MCNPX code system.

Table 2 summarizes the main irradiation characteristics (on the target back-plate over the beam spot dimensions) for SPIRAL-2 in comparison with IFMIF, ITER and DEMO [4]. In brief, SPIRAL-2 is able to provide rather comparable irradiation conditions such as ITER. It is also worth mentioning that, although neutron energy distribution of SPIRAL-2 differs from the fluxes predicted for the first wall of ITER (and DEMO) [4], the ratio of gas production over dpa rates is comparable. In the case of SPIRAL-2, we obtain He/dpa $=13, \mathrm{H} / \mathrm{dpa}=51$, while for ITER, one finds $\mathrm{He} / \mathrm{dpa}=11, \mathrm{H} / \mathrm{dpa}=45$ [4] respectively. 

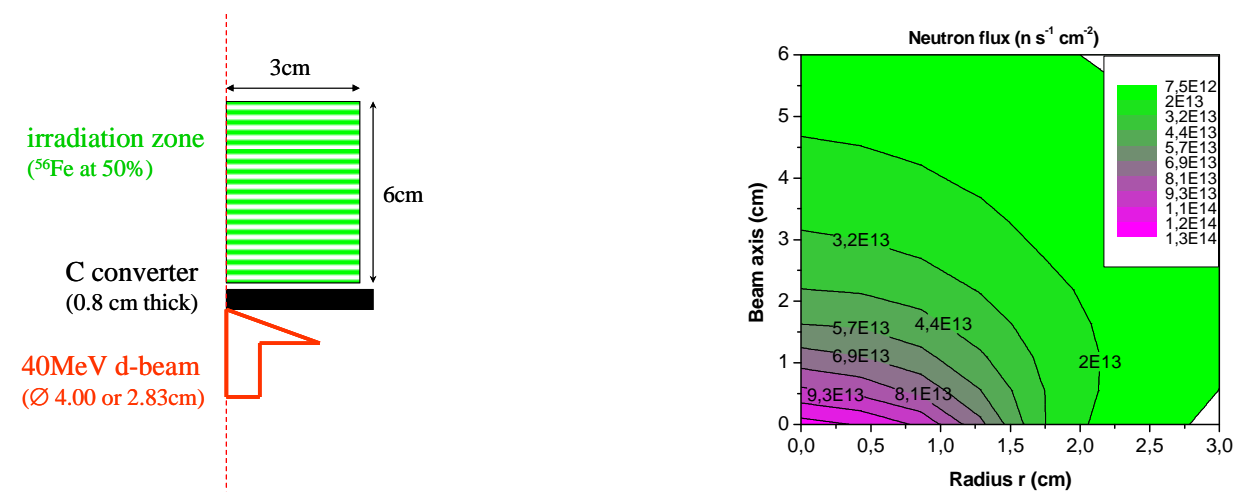

Figure 3: on the left-target geometry used for neutron flux calculations in the case of SPIRAL2; on the right - spatial distribution of the neutron flux in iron for a deuteron beam diameter of $\sim 2.83 \mathrm{~cm}$.

Table 2: Comparison of major irradiation parameters for SPIRAL-2, IFMIF, ITER and DEMO.

\begin{tabular}{|c|c|c|c|c|c|}
\hline & $\begin{array}{l}\text { Neutron } \\
\text { flux } \\
\mathrm{n} / \mathrm{s} / \mathrm{cm}^{2}\end{array}$ & $\begin{array}{c}\begin{array}{c}\text { Damage rate } \\
\text { dpa/fpy }\end{array} \\
\end{array}$ & $\begin{array}{c}\mathrm{He} \text { Gas } \\
\text { appm/fpy }\end{array}$ & $\begin{array}{c}\text { H Gas } \\
\text { appm/fpy }\end{array}$ & $\begin{array}{l}\text { Nuclear heating } \\
\text { in }{ }^{56} \mathrm{Fe} \mathrm{W} / \mathrm{cm}^{3}\end{array}$ \\
\hline IFMIF & $1.1 \times 10^{15}$ & 54 & 562 & 2622 & 23 \\
\hline SPIRAL-2 & $1.1 \times 10^{14}$ & 7 & 95 & 378 & 3 \\
\hline ITER $_{\text {max }}$ & $4.0 \times 10^{14}$ & 12 & 140 & 540 & 12 \\
\hline DEMO $_{\text {max }}$ & $1.3 \times 10^{15}$ & 30 & 320 & 1240 & 35 \\
\hline
\end{tabular}

A comparison between the different beam spot diameters assumed for SPIRAL-2 shows that a more focused deuteron beam is preferred to obtain maximal neutron fluxes. Total neutron flux attenuation is about $30 \% / \mathrm{cm}$ along z-axis. The useful irradiation volume can also be estimated: for the neutron flux (and damage rate simultaneously) larger than $5 \times 10^{13} \mathrm{n} \mathrm{s}^{-1} \mathrm{~cm}^{-2}$ (and larger than $3 \mathrm{dpa} / \mathrm{fpy}$ ), we obtain $\sim 10 \mathrm{~cm}^{3}$ and $\sim 14 \mathrm{~cm}^{3}$ for beam spot diameters of $4 \mathrm{~cm}$ and $2.83 \mathrm{~cm}$, respectively.

We also note that the irradiation temperature of the sample package has to be controlled and should be adjusted over a large range of operating temperature, say between 250 and $1000^{\circ} \mathrm{C}$. Two heat sources have to be considered: the rotating graphite converter with a temperature around $1700^{\circ} \mathrm{C}$ and nuclear heating coming from interacting neutrons and photons. Preliminary thermal calculations have shown that the temperature gradient along the beam axis could be small enough by using an additional heating around the sample container. Further detailed thermal studies, involving for example a dedicated cooling system (e.g., He-gas flow) will be necessary in order to reach a flat temperature distribution over the desired range of temperature.

For material irradiation purposes a second, similar to the RIB production plug, irradiation station should be envisaged. The UCx production target in this case could be replaced by the irradiation samples, which should be positioned as close to the converter as 
possible and a separate cooling system (e.g., He gas or water flow) has to be implemented to keep the temperature of the samples at the desired level $\left(250-1000^{\circ} \mathrm{C}\right)$. In addition, one should make sure that the sample container could be easily extracted in the shielded cell, from the entire plug after the final irradiations and certain cooling time and could be finally prepared for the transportation of irradiated material for the final tests and analysis in hot and/or chemistry external laboratories. It might be that some preliminary sample examination on-site will be performed immediately after cooling or/and in between different irradiations.

\section{Pulsed neutron beams}

The use of a neutron beam depends on specific characteristics in terms of the available neutron flux, energy resolution and useful energy range. These characteristics give strong technical constraints to the accelerator and experiment hall. As it was discussed earlier the neutrons produced by $\mathrm{d}+\mathrm{C}$ reaction present a continuous spectrum up to $\sim 40 \mathrm{MeV}$ and are peaked at forward angles. Therefore, the neutron beam should be designed at the very forward angles, presumably at $0^{\circ}$, through a channel in a thick concrete wall out from the room containing the converter (neutron source). A dedicated experimental hall should be built then right behind this wall.

Since the neutrons produced via the $\mathrm{d}+\mathrm{C}$ reaction are not mono-energetic, a pulsed mode is necessary to determine their energy by time of flight (ToF). It is well known that the energy resolution (determined by ToF) can be expressed by:

$$
\frac{\Delta E}{E}=\gamma(\gamma+1) \sqrt{\left(\frac{\Delta t}{t}\right)^{2}+\left(\frac{\Delta L}{L}\right)^{2}}
$$

with $L$ being the flight path and $\Delta L$ - the associated uncertainty; $t$ is the ToF of a neutron of energy $E$ for the distance $L ; \Delta t$ is the resolution of the time measurement and $\gamma$ is a relativity factor. $\Delta E / E$ at a given energy depends on the measurement time resolution and the distance between neutron production and physics targets. The choice of the path length is a compromise between the resolution (increasing with distance) and the available flux (decreasing with distance). Fig. 4 (on the left) shows the energy resolution for different combinations depending on the detector used. A time resolution of $8 \mathrm{~ns}$ is considered for a germanium detector while $1 \mathrm{~ns}$ is representative to a fission chamber or a scintillator coupled to a photomultiplier. It is clearly seen that energy resolution around $0.5 \%$ could be obtained in the entire energy range for $\mathrm{L}=10 \mathrm{~m}$ and would be around $1.0 \%$ for $\mathrm{L}=5 \mathrm{~m}$ when using a fast detector. For a germanium detector, in case of $(\mathrm{n}, \mathrm{xn})$ cross section measurement, a flight path of $20 \mathrm{~m}$ is necessary to ensure energy resolution better than $6 \%$ at $40 \mathrm{MeV}$.

The primary deuteron beam frequency $f$ is an important characteristic as well. If the frequency is too high, 2 events of time of flight $t$ and $t+T(T=1 / f)$ will not be discriminated. It appears that a period of $\sim 2 \mu \mathrm{s}$ is long enough to limit high $(\sim 40 \mathrm{MeV})$ and low $(\sim 1 \mathrm{MeV})$ energy neutron overlap. Therefore, the beam frequency should be limited to $f \sim 500 \mathrm{kHz}$ (or even smaller), what corresponds to a division of nearly by a factor of 200 if compared to a 
presently planned $\sim 87 \mathrm{MHz}$ repetition rate. Note that the integral neutron intensity is reduced in the same proportion. It is important to note that the beam power deposited on the converter is also decreased proportionally, making its design easier in terms of thermal conditions and damage rates.

The absolute neutron flux can now be calculated as a function of the energy in order to compare it with two major ToF facilities in Europe: n-TOF at CERN (Switzerland) and GELINA at Geel (Belgium) [5]. The following characteristics for SPIRAL-2 have been considered:

1) a $500 \mathrm{kHz}$ frequency (a reduction of a factor 200 of the SPIRAL-2 nominal intensity, i.e. $5 \mathrm{~mA} / 200$ );

2) a flight path of 5 and $10 \mathrm{~m}$.
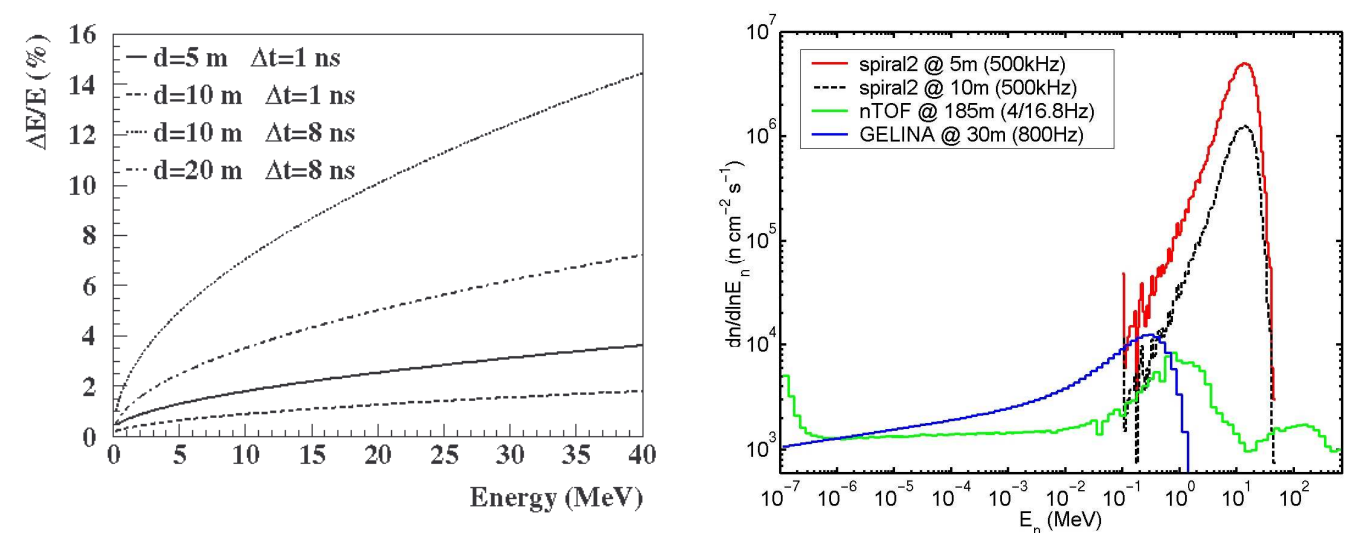

Figure 4: on the left - neutron energy resolution as a function of their energy for different combinations of flight paths $(L=5,10$ and $20 \mathrm{~m})$ and time resolutions $(\Delta t=1 \mathrm{~ns}$ and $\Delta t=8 \mathrm{~ns})$; on the right - comparison of integrated absolute neutron flux available at different ToF facilities. Data for nTOF and GELINA were taken from Ref. [6].

The results presented in Fig. 4 (on the right) show that in the range between 5 and $35 \mathrm{MeV}$ the neutron beam produced with the SPIRAL-2 is between 2 and 3 orders of magnitude higher than the flux available at n-TOF (CERN). The relatively smaller flux at the n-TOF facility is explained by a very long flight path $(\sim 185 \mathrm{~m})$ and at the same time by a very low frequency $(4 / 16.8 \mathrm{~Hz})$. Of course, one should not forget the presence of the neutron background in the experimental room due to thermal neutrons, whose flux is directly related to the averaged integral beam intensity. The low frequency of n-TOF facility leads to low average neutron flux but high signal-to-noise ratio. This ratio will be much lower at SPIRAL-2 if the entire neutron spectrum is considered. On the other hand, for fast neutrons (above $0.1 \mathrm{MeV}$ ) it should not be a limiting factor.

Another important difference for SPIRAL-2 is the expected absence of a strong accompanying gamma-flash in the beam (compared to photo-neutron sources based on electron accelerators). This feature is essential for the effective utilisation of short flight paths for the measurements discussed below. Such short flight paths are prohibited at an electron-accelerator 
based neutron source like Gelina due to the gamma-flash induced pile-up and detector disturbance.

Finally we add that the SPIRAL-2 driver could also deliver low energy deuteron beam (as low as $0.75 \mathrm{MeV} / \mathrm{A}$ ) as well as protons around below $35 \mathrm{MeV}$. These two properties would allow producing mono-energetic (or quasi-mono-energetic) neutron beams. In the first case $\left(\mathrm{E}_{\mathrm{d}}\right.$ $<4 \mathrm{MeV}$ ), mono-energetic neutrons can be produced by the ${ }^{2} \mathrm{H}(\mathrm{d}, \mathrm{n})^{3} \mathrm{He}$ reaction in the low energy range $\left(E_{n}<8 \mathrm{MeV}\right)$. In this case solid (deuterium is implemented in titanium) or gaseous targets could be used for such purposes. Higher energy neutrons (between 14 and $20 \mathrm{MeV}$ ) can also be produced in a mono-energetic way using the ${ }^{3} \mathrm{H}(\mathrm{d}, \mathrm{n})^{4} \mathrm{He}$ reaction. In this case only solid target can be used.

Quasi mono-energetic neutrons could be also produced with the proton beam by the ${ }^{7} \mathrm{Li}(\mathrm{p}, \mathrm{n})^{7} \mathrm{Be}$ reaction. The neutron energy spectrum in this case is composed of a peak around the proton energy and a large tail at lower energies. The fraction of the mono-energetic neutrons depends on the proton energy and on the lithium target thickness.

For these two applications the possible SPIRAL-2 working mode (low energy of the deuteron) is not nominal (expected incident beam current would be of the order of $1 \mathrm{~mA}$ ) but we have to keep in mind that it allows producing mono-energetic neutron beams, and the neutron flux will depend then on the beam power which could be supported by these targets. Further studies along these lines are urgently needed.

\section{Conclusions}

The decision to construct the SPIRAL-2 facility has been made in June 2005, what means that the installation should be operational around 2010. It seems that, in addition to the RIB production facility a continuous and pulsed neutron source can be envisaged using the same deuteron beam delivered by SPIRAL-2.

Both cross section measurements and various applications could be realised successfully using the high energy neutrons produced at SPIRAL-2. Two particular cases were examined in more detail, namely: (a) material activation-irradiation with high-energy high-intensity neutron fluxes, and $b$ ) neutron time-of-flight ( $\mathrm{nToF})$ measurements with pulsed neutron beams.

Thanks to the high energy and high intensity neutron flux available, SPIRAL-2 offers a unique opportunity for material irradiations both for fission and fusion related research, tests of various detection systems and of resistance of electronics components to irradiations, etc. SPIRAL-2 also could be considered as an intermediate step towards new generation dedicated irradiation facilities as IFMIF previewed only beyond $\sim 2015$.

Equally, the interval from $0.1 \mathrm{MeV}$ to $40 \mathrm{MeV}$ for neutron cross section measurements is an energy range that is of particular importance for energy applications, notably accelerator driven systems (ADS) and Gen-IV fast reactors, as well as for fusion related devices. It is also the region where pre-equilibrium approaches are often used to link the low (evaporation) and high energy (intra-nuclear cascade) reaction models. With very intense neutron beams of SPIRAL-2 measurements of very low mass (often radioactive) targets and small cross sections 
become feasible in short experimental campaigns. Production of radioactive targets for dedicated physics experiments is also an attractive feature of SPIRAL-2.

In brief, it was shown that SPIRAL-2 has got a remarkable potential for neutron based research both for fundamental physics and various applications. In addition, in the neutron energy range from a few $\mathrm{MeV}$ to, say, $35 \mathrm{MeV}$ this research would have a leading position for the next 10-15 years if compared to other neutron facilities in operation or under construction worldwide.

\section{References}

[1] Report (white paper) "LINAG Phase I", June 2002, GANIL, Caen, France; available at http://www.ganil.fr/research/developments/spiral2/index.html (March 2006).

[2] D. Ridikas et al., "Neutrons for Science (NFS) at SPIRAL-2", reports DAPNIA-03-365, DAPNIA-05-30, DAPNIA-05-40, CEA Saclay (2005); available at http://wwwdapnia.cea.fr/Documentation/Publications/index.php (March 2006); International Workshop on Neutron for Science at SPIRAL-2, 13-14 December 2004, GANIL, Caen, France; presentations and summary report available at http://spiral2ws.ganil.fr/2004/nfs/ (March 2006).

[3] L.S. Waters, "MCNPX ${ }^{T M}$ USER'S MANUAL", Los Alamos National Laboratory, preprint TPOE83-G-UG-X-00001, (November 1999), also see http://mcnpx.lanl.gov/ (March 2006).

[4] U. Fischer, "Neutronics and Nuclear Data: Status and Perspectives for Fusion Technology Applications", Proceedings of the International Workshop on Fast Neutron Physics, Dresden, Germany, 5-7 September 2002.

[5] F. Gunsing et al., "Status report of the project nTOF at CERN", report DAPNIA/SPhN CEA Saclay, Conseil Scientifique et Technique (CSTS), 16 Dec. 2002, pp.27-32; F. Gunsing, private communication. 